\section{best practice onkologie}

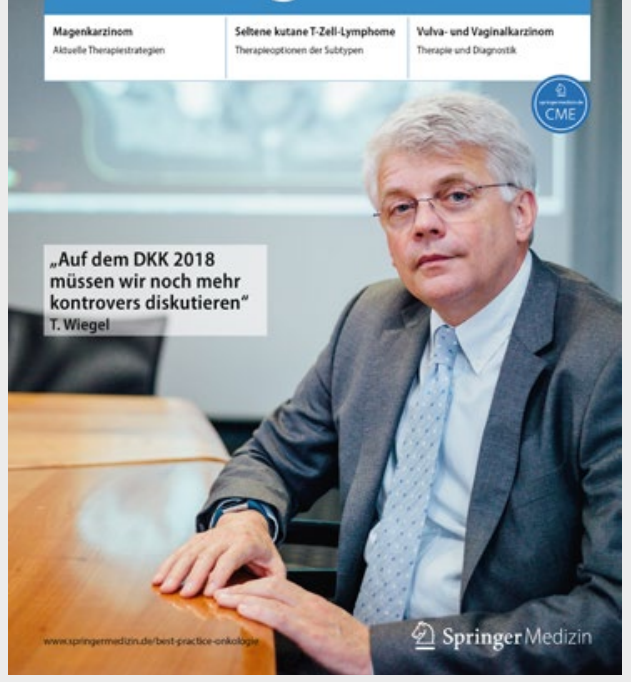

Onkologie auf den Punkt gebracht:

\section{best practice onkologie}

Die Zeitschrift best practice onkologie richtet sich an alle Ärzte, die in der onkologischen Regelversorgung tätig sind - sei es als Krebsspezialist oder als Allgemeinarzt bzw. niedergelassener Internist. Im Mittelpunkt jeder Ausgabe stehen praxisorientierte und interdisziplinäre Fortbildungsübersichten.

In der Ausgabe vom Dezember 2017 lesen Sie z.B.:

\section{Aktuelle Strategien zur Therapie des Magenkarzinoms:}

Welche kurativen Möglichkeiten sind aktuell "state-ofthe-art", und ab wann kann eine adjuvante Chemotherapie bzw. eine zielgerichtete molekulare Therapie erwogen werden?

\section{CME-Fortbildung zystische Pankreastumoren}

Welche differenzialdiagnostischen Maßnahmen sind notwendig, wie sieht das individuelle klinische Vorgehen aus?

Lesen Sie die Zeitschrift gratis online unter www.springermedizin.de/best-practice-onkologie

oder bestellen Sie Ihr kostenloses Probeabonnement per Email an:

Marketingmedizin@springer.com

Herzinsuffizienz

\title{
Versorgung in den eigenen vier Wänden
}

\author{
Müssen Patienten mit dekompensierter Herzinsuffizienz \\ wirklich so oft ins Krankenhaus wie in Deutschland? \\ Ein Beispiel aus den Niederlanden zeigt, wie es anders geht.
}

Krankenhausaufenthalte tun älteren Menschen nicht gut, das ist kein Geheimnis. Bis zu jeder sechste Patient, der über 70 sei und ins Krankenhaus müsse, entwickele innerhalb der ersten Tage ein Delir, sagte Astid van der Welde, Medical Practice Manager am Isala Heart Centre in den Niederlanden. Das hat Folgen: Um 7,5 Tage werde der Krankenhausaufenthalt dadurch im Schnitt verlängert. Die Zahl der Krankenhausinfektionen steige.

\section{Wohnzimmer statt \\ Überwachungsstation}

Bei der von der Europäischen Gesellschaft für Kardiologie unterstützten Konferenz eCardiology 2017 in Berlin berichtete die Holländerin über das seit $2009 \mathrm{im}$ Isala Heart Centre im Regelbetrieb laufende Programm Chance@Home, bei dem ältere Patienten mit dekompensierter Herzinsuffizienz und teilweise auch mit unkompliziertem akutem Myokardinfarkt nicht stationär aufgenommen, sondern in den eigenen vier Wänden versorgt werden.

Der Chance@Home Versorgung zugewiesen werden die Patienten überwiegend von den kardiologischen Stationen und Ambulanzen des Herzzentrums. Aber auch niedergelassene Allgemeinmediziner sowie Pflegeeinrichtungen können die telemedizinisch unterstützte Heimversorgung initiieren. Im Zentrum des Programms steht eine spezialisierte Heart Failure Nurse, die 24 Stunden am Tag, 7 Tage die Woche durch einen Kardiologen des Heart Centres unterstützt wird, der im Hintergrund Dienst hat und dem über ein mobiles Dokumentationssystem alle relevanten Daten zur Verfügung stehen.

Hausbesuche der Krankenpflegekraft gibt es je nach Patient täglich oder minimal alle zwei Tage. Telemetrisch überwacht werden unter anderem die Sauer- stoffsättigung, der Blutdruck und das EKG. Die Pflegekraft nimmt außerdem Laborwerte ab und kann in Absprache mit dem Kardiologen Diuretika und auch Dobutamin i.v. applizieren. Derzeit wird zusätzlich an einer Erweiterung der Point-of-Care-Labordiagnostik gearbeitet, um Doppelbesuche zu vermeiden.

\section{Kein einziges Delir innerhalb von sechs Jahren}

Insgesamt seien nach diesem Modell mittlerweile über 4000 Patienten versorgt worden, so van der Welde. Die Erfolgsquote, also der Anteil der Patienten, bei denen keine stationäre Einweisung nötig wird, liege bei rund $80 \%$. Das Durchschnittsalter steigt: Waren die in Chance@Home versorgten Patienten anfangs im Mittel Anfang 70, sind sie mittlerweile im Mittel 79 Jahre alt. Der Langzeitverlauf zeigt, dass viele Patienten über kurz oder lang sterben: „Das sind also wirklich alte, kranke Menschen“, so van der Welde. Es handle sich nicht um „junge Alte“ mit stabiler Erkrankung.

\section{Höhere Patientenzufriedenheit als bei stationärem Aufenthalt}

Evaluiert wird das Programm natürlich auch. Van der Welde berichtete, dass die Patientenzufriedenheit höher sei als bei vergleichbaren Patienten mit stationären Aufenthalten. Die Infektionsraten seien gering, die Patienten seien aktiver und stürzten seltener.

Vor allem aber: Kein einziger Patient habe seit Beginn des Programms ein Delir entwickelt. Und: „Die Versorgung ist billiger“, so van der Welde. Ein Tag in Chance@Home koste 100 bis 110 Euro, gegenüber rund 300 Euro bei einem vergleichbaren Patienten in einem niederländischen Krankenhaus. Philipp Grätzel

Kongress eCardiology 2017; Berlin,

Session "Multidisciplinary Models of Telecare" 